longitudinal associations between incarceration and later pregnancy loss in mid-adulthood (Wave V; 2016-18; ages 34-43) among women who had ever been pregnant $(n=2353)$; we estimated racial differences. Using causal mediation, we explored whether STI and PID mediated associations with pregnancy loss.

Results Incarceration was associated with a history of STI (white adjusted prevalence ratio [APR]: 1.54, 95\% CI 1.14, 2.06; Black APR: 1.26, 95\% CI 1.02, 1.56); the association between incarceration and PID was null among white women (APR: 0.99 , 95\% CI $0.47,2.09$ ) and elevated among Black women (APR: 2.82, 95\% CI 1.36, 5.83). Prior incarceration did not appear associated with pregnancy loss among white women (APR: 1.01, 95\%CI 0.70, 1.45), but was associated among Black women (APR: 1.38, 95\% CI: 0.97, 1.97), with STI appearing to partially mediate.

Conclusion Pregnancy loss may be elevated among Black women who have been incarcerated, and incarceration-related increases in STI may account for some of this association. Sexual and reproductive health care providers may want to assess history of incarceration among patients and, given the stronger associations among Black women and the higher prevalence of incarceration in this group, incarceration may have particularly deleterious consequences for their reproductive health, underscoring the need to consider alternatives to incarceration to address racial inequities.

\section{P124 SUBSTANCE ABUSE, VIOLENCE, AND SEXUAL RISK AMONG ADOLESCENT CIS-GENDER WOMEN WHO ARE AT HIGH-RISK FOR HUMAN IMMUNODEFICIENCY VIRUS}

${ }^{1} \mathrm{G}$ Vavala*, ${ }^{1} \mathrm{Q}$ Wang, ${ }^{1} \mathrm{C}$ Stafylis, ${ }^{2} \mathrm{~S}$ Jimenez, ${ }^{2} \mathrm{~W}$ Ramos, ${ }^{3} \mathrm{M}$ Ocasio, ${ }^{2} \mathrm{~A}$ Romero-Espinoza, ${ }^{4} \mathrm{R}$ Flynn, ${ }^{5} \mathrm{M}$ Fernandez, ${ }^{2} \mathrm{D}$ Swendeman, ${ }^{2} \mathrm{~W}$ Comulada, ${ }^{1} \mathrm{~J}$ Klausner. ${ }^{1}$ Division of Infectious Diseases, David Geffen School of Medicine, University of California Los Angeles, Los Angeles, USA; ${ }^{2}$ Department of Psychiatry and Biobehavioral Sciences, David Geffen School of Medicine, University of California Los Angeles, Los Angeles, USA; ${ }^{3}$ Department of Pediatrics/Section of Adolescent Medicine, Tulane University School of Medicine, New Orleans, USA; ${ }^{4}$ Los Angeles LGBT Center, Los Angeles, USA; ${ }^{5}$ Nova Southeastern University, Fort Lauderdale, USA

\subsection{6/sextrans-2021-sti.241}

Background The substance abuse, violence, and AIDS (SAVA) syndemic framework offers a holistic approach to analyze these mutually reinforcing epidemics among women with or at-risk for HIV/AIDS. We investigated the relationship between SAVA conditions and sexual risk behavior among adolescent cis-gender women.

Methods We recruited high-risk adolescents into a large prospective Adolescent Trials Network Study between May 2017 2018 in Los Angeles and New Orleans. Participants selfreported risky sexual behaviors in the past four months including condomless sex, number of sex partners, and substance use before sex. We categorized participants into having 0-3 SAVA condition(s) based on the presence or absence of self-reported substance abuse in the past 4 months, including marijuana, crack, heroine, ecstasy, and methamphetamine, childhood sexual abuse (CSA), and intimate partner violence (IPV) at baseline. We used logistic regression models to examine the association between the number of SAVA conditions and sexual risk behavior.

Results Our study included 204 cis-gender women, median age of 20 years (IQR 19-22). Overall, 80.4\% reported recent substance use, $24.5 \%$ reported recent IPV, and 39.7\% reported
CSA. Among all participants, 47.6\%, 32.4\%, and $10.8 \%$ reported occurrence of one, two, and all three SAVA condition(s), respectively. The majority of participants $(77.5 \%)$ reported condomless sex, $42.7 \%$ had more than one sex partner, and $36.8 \%$ reported substance use before sex. Each additional SAVA condition experienced by women was associated with having more than one sex partners (adjusted OR = $1.67 ; 95 \%$ confidence interval $[\mathrm{CI}]=1.17,2.40)$ and substance use before sex $(\mathrm{OR}=1.41 ; 95 \% \mathrm{CI}=0.99,2.02)$.

Conclusions In our study SAVA conditions were common. We found an association between the increased number of SAVA conditions and sexual risk behavior. How to mitigate the impact of SAVA conditions on sexual risk behavior needs further study.

\section{P125 BIOLOGICAL OUTCOME MEASUREMENT THROUGH MAIL-BASED TESTING FOR HIV, STIS, AND SUBSTANCE USE AMONG ADOLESCENTS}

${ }^{1} \mathrm{G}$ Vavala*, ${ }^{1} \mathrm{Q}$ Wang, ${ }^{1} \mathrm{C}$ Stafylis, ${ }^{2} \mathrm{~W}$ Ramos, ${ }^{3} \mathrm{M}$ Ocasio, ${ }^{2} \mathrm{~A}$ Romero-Espinoza, ${ }^{4} \mathrm{R}$ Flynn, ${ }^{5} \mathrm{M}$ Fernandez, ${ }^{2} \mathrm{D}$ Swendeman, ${ }^{2} \mathrm{~W}$ Comulada, ${ }^{1} \mathrm{~J}$ Klausner, the Adolescent Medicine Trials Network (ATN) CARES Team. 'Division of Infectious Diseases, David Geffen School of Medicine, University of California Los Angeles, Los Angeles, USA; ${ }^{2}$ Department of Psychiatry and Biobehavioral Sciences, David Geffen School of Medicine, University of California Los Angeles, Los Angeles, USA; ${ }^{3}$ Department of Pediatrics/Section of Adolescent Medicine, Tulane University School of Medicine, New Orleans, USA; ${ }^{4}$ Los Angeles LGBT Center, Los Angeles, USA; ${ }^{5}$ Nova Southeastern University, Fort Lauderdale, USA

\subsection{6/sextrans-2021-sti.242}

Background COVID-19 disrupted in-person clinical research activities. In response, the Adolescent Medicine Trials Network (ATN) CARES study instituted mail-based testing for human immunodeficiency virus (HIV), sexually transmitted infections (STIs), and substance use screening. We assessed the acceptability and feasibility of the ATN CARES mail-based testing program.

Methods From September 2020- December 2020, trained study staff sent myLAB Box $\odot$ mail-based test kits and written instructions to gay, bisexual, and transgender study participants between the ages of 16-26 years. The test kits included a dried blood spot collection card for HIV (4th generation antigen/antibody EIA) and syphilis (TP-EIA) testing, swabs and a urine collection kit for the detection of extragenital and genital Chlamydia trachomatis and Neisseria gonorrhoeae infection, and a urine collection kit for the screening of six substances. Staff tracked shipment status, and when delivery was identified, contacted participants to assist them with selfcollection and test kit return.

Results Of the 65 youth who participated to date, 49.2\% (32/ $65)$ returned their kit and completed at least one test. Those 32 participants had a median age of 23.5 years (IQR 22-25). The median number of days from test kit ordered to results was 18.5 days (IQR 15-23.5); median number of days from collection to results was 10 days (IQR 8.5-12). MyLAB Box ( reported a total of 219 test

Results 30 HIV, 22 syphilis, 56 chlamydia, 56 gonorrhea, and 55 drug tests processed. Of the 219 tests, $7.3 \%$ (16) were rejected due to specimen inadequacy (6 dried blood spot cards, 2 anal swabs, 8 urine specimens).

Conclusions Mail-based testing is a promising way to monitor a variety of biological study outcomes among adolescent study participants. Future studies should further investigate how to strengthen utilization and fidelity of mail-based testing. 